\title{
Tuberculosis: What We Don't Know Can, and Does, Hurt Us
}

\author{
David G. RusselI ${ }^{1}$, Clifton E. Barry 3rd ${ }^{2}$, and JoAnne L. Flynn ${ }^{3}$ \\ ${ }^{1}$ Department of Microbiology and Immunology College of Veterinary Medicine Cornell University \\ Ithaca, NY 14853, USA. dgr8@cornell.edu \\ ${ }^{2}$ Tuberculosis Research Section, LCID, NIAID, NIH 33 North Drive, Bldg 33 Rm 2W20D Bethesda, \\ MD, 20892, USA. cbarry@niaid.nih.gov \\ ${ }^{3}$ Department of Microbiology and Molecular Genetics University of Pittsburgh School of Medicine \\ W1144 Biomedical Science Tower Pittsburgh, PA 15261, USA. Joanne@pitt.edu
}

\begin{abstract}
Mycobacterium tuberculosis (Mtb) has a penetrance of its host population that would be the envy of most human pathogens. Approximately 1/3 of the human population is skin test positive for the infection and is thus thought to harbor the bacterium. Globally, 22 "high-burden" countries account for over $80 \%$ of the active tuberculosis cases in the world, highlighting the inequitable distribution of the disease. There is no effective vaccine against infection and current drug therapies are fraught with problems, predominantly due to the protracted nature of the treatment and the increasing occurrence of drug resistance. Here we focus on the biology of the host-pathogen interaction and discuss new and evolving strategies for intervention.
\end{abstract}

\section{Introduction}

Tuberculosis (TB) is a disease that, in the Western World, is held in check by effective public health systems that compensate for the relative shortcomings of current intervention strategies. However, due to the lack of vaccines, and the need for new, faster-acting drugs, it is unclear how the disease can ever be controlled in the countries where it is truly endemic. In addition, as highly drug-resistant strains continue to evolve we face the risk of losing control even in the industrialized world. Despite recent increases in research activity we remain hampered by significant gaps in our knowledge of the biology of this pathogen (Table 1). An increased appreciation of the bottlenecks in the life cycle of Mycobacterium tuberculosis (Mtb) should facilitate development of new intervention strategies that are applicable to those countries most in need.

\section{The Life Cycle of Mycobacterium tuberculosis}

Infection with Mtb follows a pattern of events that have been established through animal models as well as observations from human TB $(1,2)$ (Fig. 1). The infectious bacilli are inhaled as droplet nuclei that have been exhaled into the atmosphere. These droplets are small enough to remain airborne for several hours. Estimations of the minimum infectious dose range from a single bacterium upwards. Our understanding of the initial stages of infection in the lung is mainly through inference; it is generally believed that the bacteria are phagocytosed by alveolar macrophages, which then invade the subtending epithelial layer. This induces a localized inflammatory response that leads to recruitment of mononuclear cells from neighboring blood vessels, providing fresh host cells for the expanding bacterial population. These cells are the 
building blocks of the granuloma, which is the defining pathologic feature of this disease. Initially the granuloma is an amorphous mass of macrophages, monocytes and neutrophils, however, the macrophages differentiate into several specialized cell types, including multinucleated giant cells, foamy macrophages and epithelioid macrophages. With the development of an acquired immune response, and the arrival of lymphocytes, the granuloma acquires a more organized, stratified structure. The macrophage-rich center becomes surrounded by a mantle of lymphocytes that may be enclosed within a fibrous cuff that marks the periphery of the structure.

The appearance of Mtb-specific lymphocytes approximately 2-3 weeks post-infection marks the end of the phase of rapid bacterial replication and the onset of a "containment" state that, in mice, is characterized by relatively stable bacterial numbers. At this time the granuloma is extensively vascularized and cells are actively recruited to the site of infection. In granulomas exhibiting the pathology associated with disease progression, the fibrous sheath becomes more marked and the number of blood vessels that penetrate the structure diminishes. There is also an increase in the number of foamy macrophages, which may be responsible for the increase in caseous debris in the center of the granuloma (3). At these "late" stages, the caseous portion of the granuloma becomes hypoxic (4), a condition that can induce a state of non-replicative persistence in Mtb in culture. Histology of infected tissues from immunocompetent patients with active TB reveal granulomas in all states of development from containment to active disease, which implies that the fate of each granuloma is determined locally, not systemically. Active granulomas exhibit extensive pathology and, ultimately the granuloma ruptures and spills thousands of viable, infectious bacilli into the airways (5), resulting in the development of a productive cough that facilitates aerosol spread of infectious bacilli.

Recent observations in human TB patients indicate that neutrophil influx in late stage disease may also contribute to the tissue damage and the dissemination of infectious bacteria into the airways (6). Modern imaging observations on human tuberculosis stress that the balance between containment and disease progression is complex and highly dynamic, and appears to be a local phenomenon involving differential progression of individual granulomas within a single individual (7).

\section{Immune protection through vaccination}

A pre-existing, specific immune response against Mtb, acquired through vaccination or a chemotherapeutically resolved infection, has an impact on the course of TB (8) (Fig. 2). The immune host is not protected against infection, but progression to containment occurs earlier, and at a lower bacterial load. In most animal models bacterial containment is achieved at approximately 10-fold fewer bacteria in the lungs. At the human population level this would be significant because progression to disease is linked directly to bacterial load. If disease is determined at the level of the individual granuloma, then the presence of fewer granulomas may reduce the chance of developing active disease.

Although the contribution of antimicrobial effectors has been established in mice $(9,10)$, the relative hierarchy of immune-mediated killing mechanisms in humans is unclear. However, from the increased susceptibility of $\mathrm{HIV}^{+}$humans, we infer that CD4 $\mathrm{T}$ cells are important in the control of human tuberculosis. Similarly, the use of tumor necrosis factor (TNF)neutralizing agents for treatment of inflammatory diseases substantially increases the risk of tuberculosis, which suggests that the level of this cytokine is critical to the balance between disease control and pathology (11-14). From these data, and studies of genetic mutations that predispose humans to tuberculosis (15), we infer that, similar to mice, macrophage activation in humans is central to the control of infection. 


\section{BCG: It's not for everyone}

Bacillus Calmette-Guérin (BCG) is the only approved vaccine against TB. It was developed though the serial in vitro passage of Mycobacterium bovis until it became nonpathogenic. It is used in countries with endemic TB because it protects children against severe forms of disease, such as TB meningitis or disseminated infection. However, although effective against development of TB in some countries such as the UK (16), its efficacy has been questioned in several studies, most notably in India, where very limited (or no) protection has been reported (17). There are three main hypotheses as to why BCG works in some populations but not in others. First, BCG has become too attenuated through culture and modern preparations of the vaccine are too benign to generate adequate protective immunity (18). Second, exposure of infants to environmental mycobacteria in countries like India could lead to tolerance (19-21) or, third, clearance of the BCG in some populations may occur prior to development of a protective immune response. Clearly, as we move forward with new vaccine constructs it is vital that we better understand the limitations of BCG-induced protection so that a new vaccine can be effective in those countries where it is most needed.

New anti-TB vaccination strategies can be divided into three broad categories. First, improving BCG by adding or over-expressing strongly immunogenic Mtb antigens, thus enhancing and broadening the immune responses induced by the recombinant bacterium (22-24). Second, attenuating strains of Mtb through the deletion of genes for specific metabolic pathways required for survival or full virulence (25-27). And third, the use of prime-boost strategies that direct and amplify an initial "protective" immune response through subsequent inoculation with viral vectors encoding Mtb antigens or protein subunits (28-33).

However, if the protective immune response to these strains is subject to the same environmental factors that limit the effectiveness of BCG in humans then we have not progressed. This may, however, be circumvented through the prime-boost strategy of supplementing vaccination with BCG, or recombinant strains, with non-replicating adenovirus or vaccinia virus strains encoding Mtb antigens (28-33), which could supplement existing BCG vaccination programs, although this too has met with mixed results $(30,32)$.

\section{Transition into the field}

So, given the limited efficacy of immune protection, where would vaccines be most useful in combating TB? Vaccination could play an important role in reducing transmission in areas that have a low incidence of HIV, particularly if there are multi-drug resistant (MDR) strains present. However, the impact of vaccination on populations with high incidence of HIV, such as sub-Saharan Africa, is open to question. Transmission of TB appears to be less efficient in HIV patients because a robust immune response is a significant contributor to the pathology required for transmission (34-36). However in areas where the co-incidence of HIV and Mtb infections exceeds $80 \%$ of TB cases, this is unlikely to have a discernable impact. We have yet to see if these new modified vaccine strains will improve the protection afforded by BCG or will recapitulate the absence of efficacy of BCG vaccination amongst ethnic groups most at risk from the disease. The changing landscape of circulating hypervirulent strains, such as the immunosuppressive Beijing lineage, which may have emerged in response to BCG vaccination, also interjects some caution in extrapolation from results generated in the laboratory $(37,38)$.

\section{Chemotherapeutic intervention}

Current chemotherapeutic regimens require more than 6 months treatment with multiple drugs. Such regimens are plagued with issues of patient non-compliance, inadequate health care oversight, and the increasing proliferation of drug-resistant strains. The need for fast-acting, effective medications for use in resource-poor countries is immediate. 
The balance of the physiological state(s) of the bacterial population inside the granuloma during the dynamic alterations in its structure is clearly central to persistence and progression to disease. Research involving bacterial strains defective in genes critical to various metabolic pathways has revealed different defects during the course of the infection cycle in mice. This implies, not surprisingly, that the bacterium regulates its metabolism differentially during the progression of disease (7). Such metabolic shifts could be driven by the availability of certain nutrients or carbon sources, the aerobic or hypoxic nature of the granuloma, or the level of stress induced by various infected host cells and the immune response. Because of the variability between granulomas within a single infected individual, and their highly differentiated internal structure, granulomas offer an extremely diverse range of environments for Mtb.

In a contained lesion, the bacterial load appears relatively static and it has been hypothesized that the bacteria are in a non-replicative state of vegetative metabolism $(39,40)$. However, more recent work monitoring bacterial replication via an unstable "clock" plasmid indicates that the bacterial population, even in apparently stable lesions, continues to undergo replication (41). It is unlikely that there is a simple binary distribution of bacteria between replicating and nonreplicating status, and even within a single granuloma there are likely to be multiple local microenvironments supporting unique bacterial populations. The drugs that are currently in common usage preferentially target replicating organisms, and therefore a non-replicative subpopulation of the bacteria present in the persistent or "latent" infection will show innate resistance to drugs. The innate resistance of non-replicating Mtb may contribute significantly to the protracted treatment period required in current therapeutic regimens.

\section{Bacterial metabolism during infection}

Whilst the murine model for TB is imperfect, it has proven to be an extremely useful tool in probing some of the metabolic shifts required to sustain a bacterium transitioning from rich broth into an in vivo infection $(42,43)$.

One good example of a metabolic shift required to support infection is the realignment of the bacterium's lipid metabolism. Isocitrate lyase activity is required to sustain Mtb infection in the chronic phase of infection in the murine model (44). The enzyme appears to fulfill a detoxification function, controlling propionate levels when the bacterium uses specific carbon sources such as cholesterol (45-49), which has been invoked as a nutrient during the persistent phase of infection $(46,50)$.

Recent studies with the hypoxia indicator pimonidazole, coupled with direct measurements via oxygen probe in lesions of primate and rabbit models, has confirmed previous speculation that the inner regions of a fully stratified granuloma have extremely low, but detectable oxygen tension (4). This has implications for tissue pathology but it may also affect the metabolic state of the bacterium, which upregulates the DosR regulon to promote survival and re-emergence into the growth state (51).

\section{Drug discovery programs: Introduction of realism at the level of screening}

Drug discovery programs for the identification of novel targets as well as new antimicrobials are few and far between. The more traditional methods of target-based discovery and remodeling of known scaffolds have pretty much exhausted known possibilities. Antiinfectives as a field has placed an inappropriate emphasis on the need to demonstrate genetic "essentiality" for any target, which is based on the misplaced assumption that genetic knockouts can be phenotypically copied by small molecule inhibitors of metabolic pathways. We need to shift our mindset from the discovery of essential gene products to appreciating the subtle 
holistic interactions between pathways and gene products that are required for survival in the many different microenvironments of the host.

Increasingly sophisticated cell-based screens of Mtb in a variety of conditions that simulate the multiple microenvironments of the host are needed to identify those metabolic breakpoints that could feed new target-based development programs. Both from the basic and the translational science aspects, understanding the adaptation of the organism and its points of vulnerability under various environmental conditions represents a logical pathway towards agents potent within the granuloma that may shorten therapy. Just as important is a better understanding of the microenvironments experienced by the bacterium in the human host, so that these can be replicated in vitro. As one example, screening directly on Mtb-infected macrophages in differing states of activation would incorporate many of the host-mediated pressures responsible for inducing the different replicative and non-replicative states of $\mathrm{Mtb}$ observed in the human host (52).

\section{Missing tools}

As the field moves forward to evaluate new drugs and vaccines we are clearly lacking some of the most basic tools for assessing efficacy. The mouse model fails to form granulomas that reproduce the tissue environments seen in humans. Accurate reproduction of these environments is likely to be important to both chemo- and immunotherapy. Rabbits and guinea pigs generate a tissue response that appears more comparable to humans, but still requires validation in both human and primate infections $(4,53-56)$. Rigorous studies comparing the disease in animal models to that of human disease are crucial to defining the validity of each model system.

The second critical shortcoming in our portfolio is the complete absence of any biomarkers for disease status (57). In mice, one can isolate tissues and count colony-forming units to determine the progression of disease in the context of bacterial replication but we cannot do this in humans and there is no simple, non-invasive means of assaying whether an individual is containing their infection or progressing to active disease. The diagnostic tests in common usage today merely measure whether or not an individual has mounted an immune response to Mtb. Given that progression to disease is determined locally, at the level of the individual granuloma, it is unclear if we will ever be able to develop biomarkers for disease progression based on systemic readouts of immune status (58). Recent advances in PET and CT-based imaging modalities have the potential to fill this gap (Fig. 4), but the relationship between the observed pathology and bacterial burden has yet to be established. Because such imaging is not a practical method for diagnosing TB in the field, studies correlating comparable imaging data with immune responses or metabolites measurable in readily obtainable human samples (blood, urine) should be undertaken in an attempt to identify possible biomarkers of infection and disease. This is an extremely serious gap in moving new therapies into the field where, currently, impact becomes apparent only after many years.

\section{Conclusions, and the cold slap of reality}

The development of new drugs and vaccines are sorely needed but they are not the complete answer. Tuberculosis is an infection that can be held in check by an effective Public Health system. Failure of the local healthcare system can lead to the systematic selection and spread of drug resistant strains, as recently observed in the Kwazulu-Natal province of South Africa, where extensively drug resistant (XDR) strains resistant to as many as 7 drugs are being observed (59-61). During the period leading up to the identification of these XDR strains the health care program in this region was in disarray with only $18 \%$ of sputum positive and $29 \%$ of hospitalized TB patients completing their treatment regimen. The coincidence of HIV was $80 \%$ in TB patients in this region, and there was no testing for drug-resistance conducted on 
the Mtb strains from patients. It is postulated that failed treatment regimens, in combination with the absence of drug resistance testing, led to the selection and spread of these lethal strains. The importance of an appropriately resourced, effectively managed public health system cannot be overstated. Even the best drugs or vaccines in the world will fail without an effective healthcare infrastructure to administer and monitor their application. It is only through the combination of drugs, vaccines, and public health surveillance that we can hope to break the cycle of transmission, whereby each TB patient is responsible for one or more new cases of active disease.

\section{Acknowledgments}

Research in the authors' laboratories is supported by NIH grants AI067027, AI057086, AI080651, HL055936, HL100928 (DGR) and AI50732, HL71241, HL092883 and HL075845 (JLF) and by the Intramural Research Program of the NIAID, NIH (CEB). All three authors receive support from the Bill and Melinda Gates Foundation.

\section{References and notes}

1. Flynn JL, Chan J. What's good for the host is good for the bug. Trends Microbiol Mar;2005 13:98. [PubMed: 15737727]

2. Russell DG. Who puts the tubercle in tuberculosis? Nat Rev Microbiol Jan;2007 5:39. [PubMed: 17160001]

3. Russell DG, Cardona PJ, Kim MJ, Allain S, Altare F. Foamy macrophages and the progression of the human tuberculosis granuloma. Nat Immunol Sep;2009 10:943. [PubMed: 19692995]

4. Via LE, et al. Tuberculous granulomas are hypoxic in guinea pigs, rabbits, and nonhuman primates. Infect Immun Jun;2008 76:2333. [PubMed: 18347040]

5. Kaplan G, et al. Mycobacterium tuberculosis growth at the cavity surface: a microenvironment with failed immunity. Infect Immun Dec;2003 71:7099. [PubMed: 14638800]

6. Eum SY, et al. Neutrophils are the predominant infected phagocytic cells in the airways of patients with active pulmonary TB. Chest Jan;137:122. [PubMed: 19749004]

7. Barry CE 3rd, et al. The spectrum of latent tuberculosis: rethinking the biology and intervention strategies. Nat Rev Microbiol Dec;2009 7:845. [PubMed: 19855401]

8. Dietrich J, Doherty TM. Interaction of Mycobacterium tuberculosis with the host: consequences for vaccine development. Apmis May;2009 117:440. [PubMed: 19400867]

9. MacMicking JD, et al. Altered responses to bacterial infection and endotoxic shock in mice lacking inducible nitric oxide synthase. Cell May 19;1995 81:641. [PubMed: 7538909]

10. Nathan C. Role of iNOS in human host defense. Science Jun 30;2006 312:1874. [PubMed: 16809512]

11. Bekker LG, et al. Immunopathologic effects of tumor necrosis factor alpha in murine mycobacterial infection are dose dependent. Infect Immun Dec;2000 68:6954. [PubMed: 11083819]

12. Lin PL, et al. Tumor necrosis factor neutralization results in disseminated disease in acute and latent Mycobacterium tuberculosis infection with normal granuloma structure in a cynomolgus macaque model. Arthritis Rheum Jan 7;62:340. [PubMed: 20112395]

13. Plessner HL, et al. Neutralization of tumor necrosis factor (TNF) by antibody but not TNF receptor fusion molecule exacerbates chronic murine tuberculosis. J Infect Dis Jun 1;2007 195:1643. [PubMed: 17471434]

14. Tsenova L, Bergtold A, Freedman VH, Young RA, Kaplan G. Tumor necrosis factor alpha is a determinant of pathogenesis and disease progression in mycobacterial infection in the central nervous system. Proc Natl Acad Sci U S A May 11;1999 96:5657. [PubMed: 10318940]

15. Fortin A, Abel L, Casanova JL, Gros P. Host genetics of mycobacterial diseases in mice and men: forward genetic studies of BCG-osis and tuberculosis. Annu Rev Genomics Hum Genet 2007;8:163. [PubMed: 17492906]

16. Black GF, et al. BCG-induced increase in interferon-gamma response to mycobacterial antigens and efficacy of BCG vaccination in Malawi and the UK: two randomised controlled studies. Lancet Apr 20;2002 359:1393. [PubMed: 11978337] 
17. Verma I, Grover A. Antituberculous vaccine development: a perspective for the endemic world. Expert Rev Vaccines Nov;2009 8:1547. [PubMed: 19863247]

18. Behr MA, Small PM. Has BCG attenuated to impotence? Nature Sep 11;1997 389:133. [PubMed: 9296487]

19. Brandt L, et al. Failure of the Mycobacterium bovis BCG vaccine: some species of environmental mycobacteria block multiplication of BCG and induction of protective immunity to tuberculosis. Infect Immun Feb;2002 70:672. [PubMed: 11796598]

20. Lozes E, et al. Cross-reactive immune responses against Mycobacterium bovis BCG in mice infected with non-tuberculous mycobacteria belonging to the MAIS-Group. Scand J Immunol Jul;1997 46:16. [PubMed: 9246204]

21. Demangel C, Garnier T, Rosenkrands I, Cole ST. Differential effects of prior exposure to environmental mycobacteria on vaccination with Mycobacterium bovis BCG or a recombinant BCG strain expressing RD1 antigens. Infect Immun Apr;2005 73:2190. [PubMed: 15784562]

22. Horwitz MA. Recombinant BCG expressing Mycobacterium tuberculosis major extracellular proteins. Microbes Infect May;2005 7:947. [PubMed: 15919223]

23. Williams A, et al. Evaluation of vaccines in the EU TB Vaccine Cluster using a guinea pig aerosol infection model of tuberculosis. Tuberculosis (Edinb) Jan-Mar;2005 85:29. [PubMed: 15687025]

24. Grode L, et al. Increased vaccine efficacy against tuberculosis of recombinant Mycobacterium bovis bacille Calmette-Guerin mutants that secrete listeriolysin. J Clin Invest Sep;2005 115:2472. [PubMed: 16110326]

25. Aguilar D, et al. Immunological responses and protective immunity against tuberculosis conferred by vaccination of Balb/C mice with the attenuated Mycobacterium tuberculosis (phoP) SO2 strain. Clin Exp Immunol Feb;2007 147:330. [PubMed: 17223975]

26. Hinchey J, et al. Enhanced priming of adaptive immunity by a proapoptotic mutant of Mycobacterium tuberculosis. J Clin Invest Aug;2007 117:2279. [PubMed: 17671656]

27. Waters WR, et al. Failure of a Mycobacterium tuberculosis DeltaRD1 DeltapanCD double deletion mutant in a neonatal calf aerosol M. bovis challenge model: comparisons to responses elicited by $\mathrm{M}$. bovis bacille Calmette Guerin. Vaccine Nov 7;2007 25:7832. [PubMed: 17931755]

28. Elvang T, et al. CD4 and CD8 T cell responses to the M. tuberculosis Ag85BTB10.4 promoted by adjuvanted subunit, adenovector or heterologous prime boost vaccination. PLoS One 2009;4:e5139. [PubMed: 19357780]

29. Skeiky YA, et al. Non-clinical efficacy and safety of HyVac4:IC31 vaccine administered in a BCG prime-boost regimen. Vaccine. Nov 5;2009

30. Tchilian EZ, et al. Immunogenicity and protective efficacy of prime-boost regimens with recombinant (delta)ureC hly+ Mycobacterium bovis BCG and modified vaccinia virus ankara expressing $\mathrm{M}$. tuberculosis antigen 85A against murine tuberculosis. Infect Immun Feb;2009 77:622. [PubMed: 19064635]

31. Vordermeier HM, et al. Viral booster vaccines improve Mycobacterium bovis BCG-induced protection against bovine tuberculosis. Infect Immun Aug;2009 77:3364. [PubMed: 19487476]

32. Whelan KT, et al. Safety and immunogenicity of boosting BCG vaccinated subjects with BCG: comparison with boosting with a new TB vaccine, MVA85A. PLoS One 2009;4:e5934. [PubMed: 19529780]

33. Reed SG, et al. Defined tuberculosis vaccine, Mtb72F/AS02A, evidence of protection in cynomolgus monkeys. Proc Natl Acad Sci U S A Feb 17;2009 106:2301. [PubMed: 19188599]

34. de Noronha AL, Bafica A, Nogueira L, Barral A, Barral-Netto M. Lung granulomas from Mycobacterium tuberculosis/HIV-1 co-infected patients display decreased in situ TNF production. Pathol Res Pract 2008;204:155. [PubMed: 18096327]

35. Doenhoff MJ. Granulomatous inflammation and the transmission of infection: schistosomiasis--and TB too? Immunol Today Oct;1998 19:462. [PubMed: 9785670]

36. Ledru E, Ledru S, Zoubga A. Granuloma formation and tuberculosis transmission in HIV-infected patients. Immunol Today Jul;1999 20:336. [PubMed: 10379053]

37. Parwati I, van Crevel R, van Soolingen D. Possible underlying mechanisms for successful emergence of the Mycobacterium tuberculosis Beijing genotype strains. Lancet Infect Dis Feb;10:103. [PubMed: 20113979] 
38. Shamputa IC, et al. Genetic diversity of Mycobacterium tuberculosis isolates from a tertiary care tuberculosis hospital in South Korea. J Clin Microbiol Feb;48:387. [PubMed: 20018816]

39. Munoz-Elias EJ, et al. Replication dynamics of Mycobacterium tuberculosis in chronically infected mice. Infect Immun Jan;2005 73:546. [PubMed: 15618194]

40. Rees RJ, Hart PD. Analysis of the host-parasite equilibrium in chronic murine tuberculosis by total and viable bacillary counts. Br J Exp Pathol Feb;1961 42:83. [PubMed: 13740304]

41. Gill WP, et al. A replication clock for Mycobacterium tuberculosis. Nat Med Feb;2009 15:211. [PubMed: 19182798]

42. Sassetti CM, Boyd DH, Rubin EJ. Comprehensive identification of conditionally essential genes in mycobacteria. Proc Natl Acad Sci U S A Oct 23;2001 98:12712. [PubMed: 11606763]

43. Sassetti CM, Rubin EJ. Genetic requirements for mycobacterial survival during infection. Proc Natl Acad Sci U S A Oct 28;2003 100:12989. [PubMed: 14569030]

44. McKinney JD, et al. Persistence of Mycobacterium tuberculosis in macrophages and mice requires the glyoxylate shunt enzyme isocitrate lyase. Nature Aug 17;2000 406:735. [PubMed: 10963599]

45. Munoz-Elias EJ, Upton AM, Cherian J, McKinney JD. Role of the methylcitrate cycle in Mycobacterium tuberculosis metabolism, intracellular growth, and virulence. Mol Microbiol Jun; 2006 60:1109. [PubMed: 16689789]

46. Pandey AK, Sassetti CM. Mycobacterial persistence requires the utilization of host cholesterol. Proc Natl Acad Sci U S A Mar 18;2008 105:4376. [PubMed: 18334639]

47. Upton AM, McKinney JD. Role of the methylcitrate cycle in propionate metabolism and detoxification in Mycobacterium smegmatis. Microbiology Dec;2007 153:3973. [PubMed: 18048912]

48. Van der Geize R, et al. A gene cluster encoding cholesterol catabolism in a soil actinomycete provides insight into Mycobacterium tuberculosis survival in macrophages. Proc Natl Acad Sci U S A Feb 6;2007 104:1947. [PubMed: 17264217]

49. Yang X, Nesbitt NM, Dubnau E, Smith I, Sampson NS. Cholesterol metabolism increases the metabolic pool of propionate in Mycobacterium tuberculosis. Biochemistry May 12;2009 48:3819. [PubMed: 19364125]

50. Peyron $P$, et al. Foamy macrophages from tuberculous patients' granulomas constitute a nutrient-rich reservoir for M. tuberculosis persistence. PLoS Pathog Nov;2008 4:e1000204. [PubMed: 19002241]

51. Rustad TR, Sherrid AM, Minch KJ, Sherman DR. Hypoxia: a window into Mycobacterium tuberculosis latency. Cell Microbiol Aug;2009 11:1151. [PubMed: 19388905]

52. Christophe T, et al. High content screening identifies decaprenyl-phosphoribose 2' epimerase as a target for intracellular antimycobacterial inhibitors. PLoS Pathog Oct;2009 5:e1000645. [PubMed: 19876393]

53. Lin PL, et al. Early events in Mycobacterium tuberculosis infection in cynomolgus macaques. Infect Immun Jul;2006 74:3790. [PubMed: 16790751]

54. Lin PL, et al. Quantitative comparison of active and latent tuberculosis in the cynomolgus macaque model. Infect Immun Oct;2009 77:4631. [PubMed: 19620341]

55. Ly LH, McMurray DN. The Yin-Yang of TNFalpha in the guinea pig model of tuberculosis. Indian J Exp Biol Jun;2009 47:432. [PubMed: 19634707]

56. Manabe YC, et al. The aerosol rabbit model of TB latency, reactivation and immune reconstitution inflammatory syndrome. Tuberculosis (Edinb) May;2008 88:187. [PubMed: 18068491]

57. Doherty M, Wallis RS, Zumla A. Biomarkers for tuberculosis disease status and diagnosis. Curr Opin Pulm Med May;2009 15:181. [PubMed: 19396972]

58. Doherty TM, Wallis RS, Zumla A. Biomarkers of disease activity, cure, and relapse in tuberculosis. Clin Chest Med Dec;2009 30:783. [PubMed: 19925967]

59. Loveday M, Thomson L, Chopra M, Ndlela Z. A health systems assessment of the KwaZulu-Natal tuberculosis programme in the context of increasing drug resistance. Int J Tuberc Lung Dis Sep;2008 12:1042. [PubMed: 18713502]

60. Jones KD, Hesketh T, Yudkin J. Extensively drug-resistant tuberculosis in sub-Saharan Africa: an emerging public-health concern. Trans R Soc Trop Med Hyg Mar;2008 102:219. [PubMed: 18243260] 
61. Pillay M, Sturm AW. Evolution of the extensively drug-resistant F15/LAM4/KZN strain of Mycobacterium tuberculosis in KwaZulu-Natal, South Africa. Clin Infect Dis Dec 1;2007 45:1409. [PubMed: 17990220] 

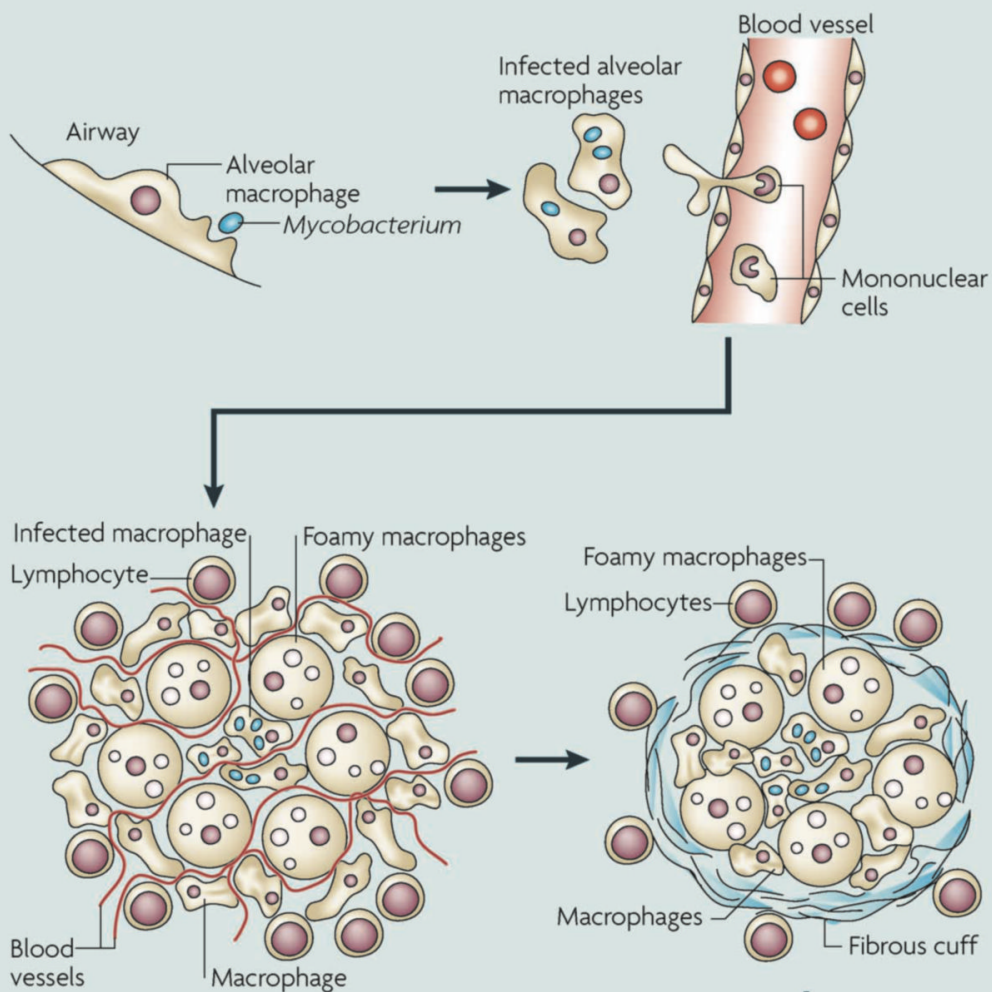

vessels
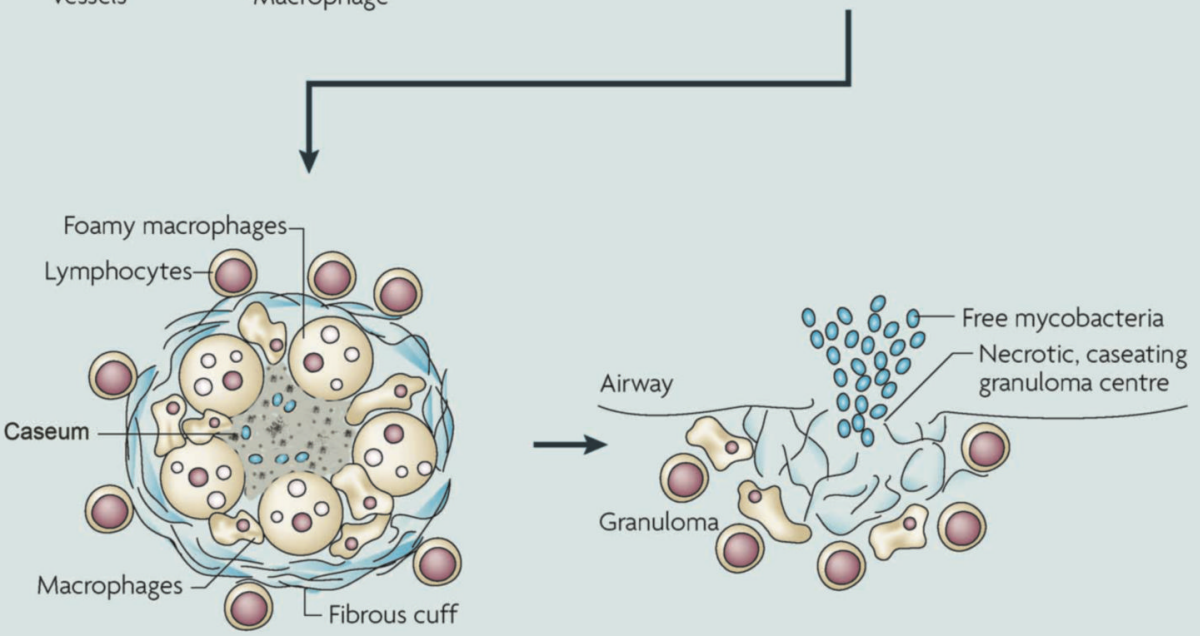

Figure 1. The life cycle of Mycobacterium tuberculosis

The infection is initiated when Mtb, present in exhaled droplets or nuclei, are inhaled and phagocytosed by resident alveolar macrophages. The resulting pro-inflammatory response triggers the infected cells to invade the subtending epithelium. This response also leads to the recruitment of monocytes from the circulation and extensive neovascularization of the infection site. The macrophages in the granulomas differentiate to form epithelioid cells, multi-nucleate giant cells and foam cells filled with lipid droplets. The granuloma can become further stratified by the formation of a fibrous cuff of extracellular matrix material that is laid down outside the macrophage layer. Lymphocytes appear to be restricted primarily to this peripheral area. Many of the granulomas persist in this balanced state, but progression towards disease is characterized 
by the loss of vascularization, the increased necrosis and the accumulation of caseum in the granuloma center. Ultimately, infectious bacilli are released into the airways when the granuloma cavitates and collapses into the lungs. 


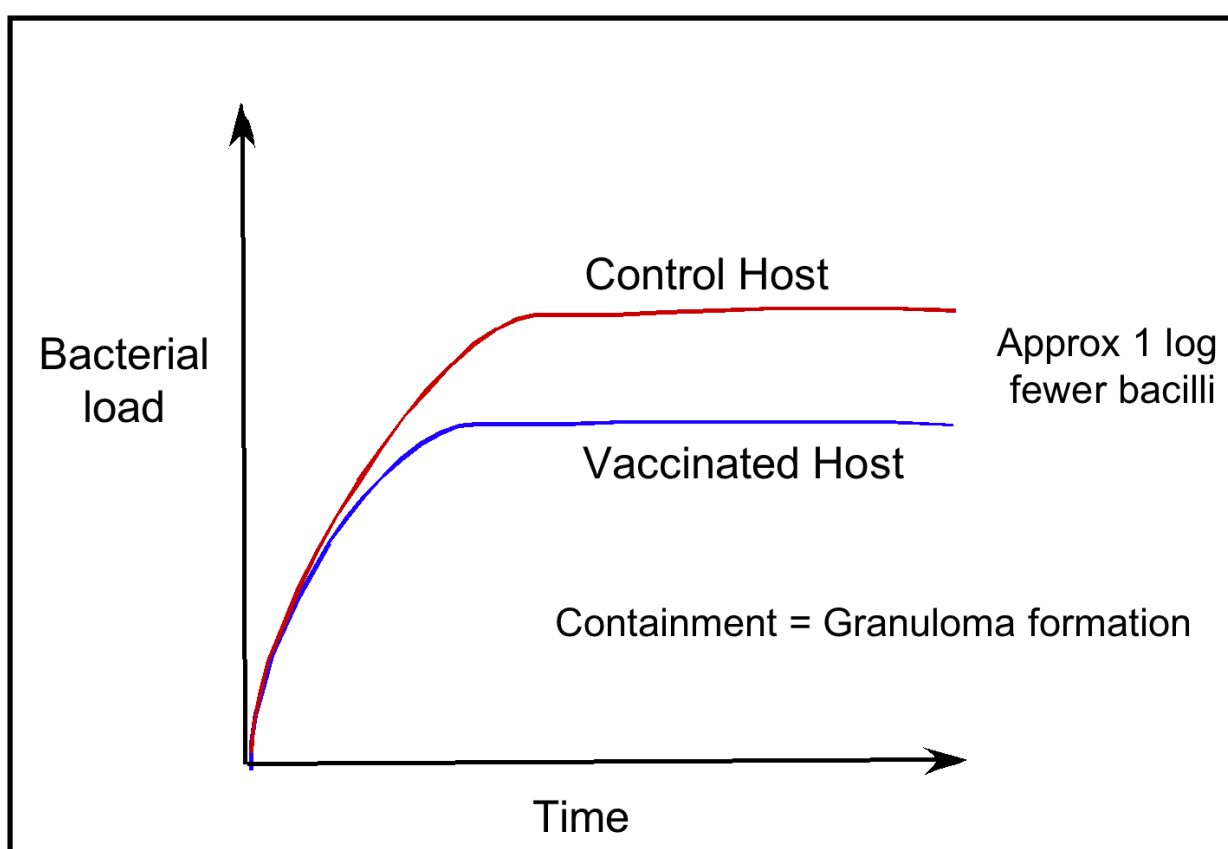

Figure 2. The Bacterial Load in Naïve and Immune Hosts

The course of Mtb infection in naïve and immune hosts follows a reproducible pattern in the mouse model. Following infection the bacteria replicate exponentially for a period of time until the host develops an acquired immune response, which limits bacterial replication. At this time the infection transitions to a state of persistence or chronicity where the bacterial load is sustained at a relatively constant level. Transition into this persistent state occurs earlier in a vaccinated host, or a host that has been infected and treated chemotherapeutically. In mice vaccinated with BCG this transition occurs with a bacterial load that is approximately 10 fold fewer than naïve host. The transition into persistence is marked by the development of a granuloma. 

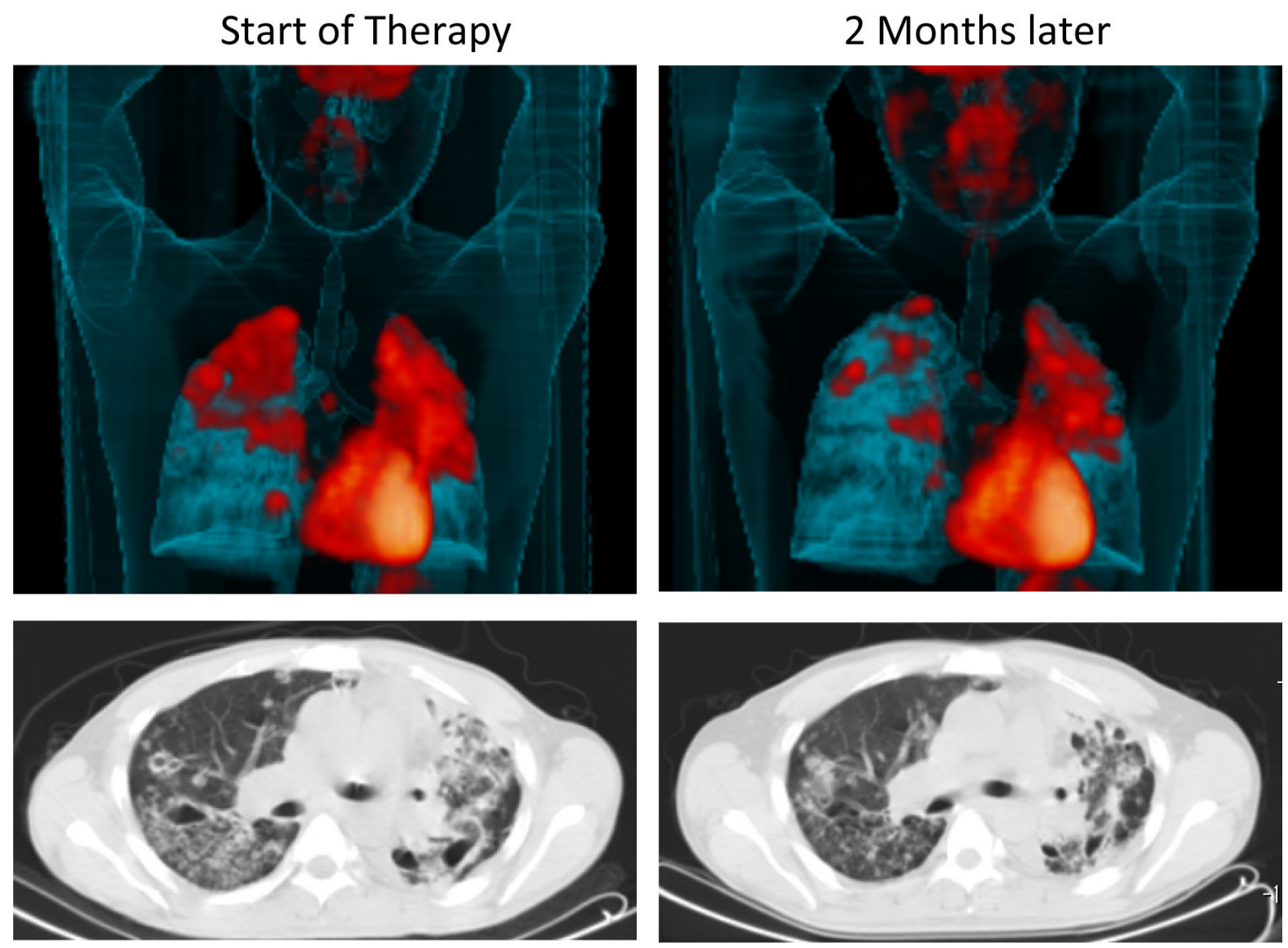

Figure 3. Imaging disease progression in humans

${ }^{18}$ F-FDG-PET/CT scan from a patient with pulmonary TB at the time when therapy was initiated (left panels) and 2 months after treatment has begun (right panels). Below are two matched axial CT slices from the area indicated by the plane on the left taken from the same scans. FDG is primarily a marker of increased tissue metabolism such as inflammation while the CT shows the structural abnormalities within this patient's lungs. Both sets of images illustrate the wide range of lesion types occupied by Mtb during infection that show differential kinetics of response to chemotherapy. 
Table 1

\begin{tabular}{|c|c|}
\hline Global Issue & Underlying Regional Problems \\
\hline $\begin{array}{l}\text { Failure to provide consistent } \\
\text { infrastructural support for healthcare }\end{array}$ & $\begin{array}{ll}\text { - } & \text { Inadequate public health sector in many regions } \\
\text { - } & \text { Variation in disease manifestations across individuals }\end{array}$ \\
\hline $\begin{array}{l}\text { Failure to develop effective diagnostic and } \\
\text { disease status indicators (biomarkers). }\end{array}$ & 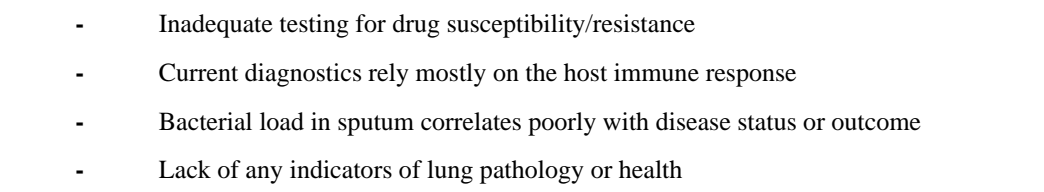 \\
\hline $\begin{array}{l}\text { Failure of BCG vaccination to protect } \\
\text { adult pulmonary TB }\end{array}$ & $\begin{array}{ll}\text { - } & \text { Variability in host response to vaccination } \\
\text { - } & \text { Variability between BCG vaccine strains } \\
\text { - } & \text { Variability in bacterial strains causing infection } \\
\text { - } & \text { Variability in host genetics/environment }\end{array}$ \\
\hline $\begin{array}{l}\text { Failure of chemotherapy to cure many } \\
\text { patients with TB }\end{array}$ & $\begin{array}{ll}\text { - } & \text { Length of therapy makes compliance difficult } \\
\text { - } & \text { Individual lesion types respond at different rates } \\
\text { - } & \text { Subpopulations of drug-tolerant bacteria } \\
\text { - } & \text { Constant emergence of new drug resistant strains }\end{array}$ \\
\hline $\begin{array}{l}\text { Failure of target-based drug discovery } \\
\text { programs }\end{array}$ & 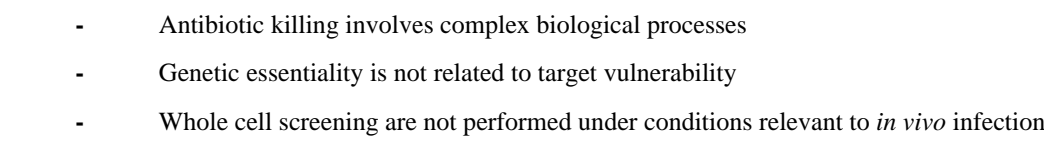 \\
\hline
\end{tabular}

